\title{
Grating and plasmon resonances in the scattering of light by finite silver nanostrip gratings
}

\author{
O.V. Shapoval ${ }^{1}$, R. Sauleau ${ }^{2}$, A.I. Nosich ${ }^{1}$ \\ ${ }^{1}$ Institute of Radio-Physics and Electronics, NAS of Ukraine, \\ 12, Proskury str., 61085 Kharkiv, Ukraine \\ E-mail: olga.v.shapoval@gmail.com \\ ${ }^{2}$ IETR, University of Rennes 1, Campus Beaulieu, bat 11-D, 35042 Rennes Cedex, France \\ E-mail: ronan.sauleau@univ-rennes1.fr
}

\begin{abstract}
We study numerically the H-polarized wave scattering by finite flat gratings of $N$ silver nanostrips in free space in the context of co-existence of surface plasmon resonances (SPR) and periodicity-induced grating resonances (GRs). The accurate numerical analysis is carried out using the previously developed combination of two-side generalized boundary conditions imposed on the strip median lines and Nystrom-type discretization of the relevant singular and hyper-singular integral equations. Our computations are focused on specific periodicity-caused coupling which leads to the existence of the grating or lattice resonances near to $\lambda_{G}=p / m, m=1,2, \ldots$ (at normal incidence). These resonances result in large reflection, transmission, absorption, and near-field enhancement. We also study the interplay of SPR and GR, if they approach each other and the optical response dependence of the grating parameters, such as overall dimension and number of strips.
\end{abstract}

Keywords: finite nanostrip grating, surface plasmon resonance, grating resonance, twoside generalized boundary conditions, singular and hyper-singular integral equations, Nystrom-type discretization.

Manuscript received 08.05.12; revised version received 07.06.12; accepted for publication 14.06.12; published online 25.09.12.

\section{Introduction}

Optical nanoantennas have unique ability of concentrating the light within nanoscale domains thanks to plasmon resonances (PRs), as revealed by measuring or computing the scattering and absorption of light by noble-metal scatterers. In this connection, noble-metal nanosize strips and their finite ensembles are very attractive as easily manufactured components of various optical devices $[1,2]$. The typical dimensions of metal nanostrips are: the width from 100 to $1000 \mathrm{~nm}$ and the thickness from 5 to $50 \mathrm{~nm}$. Thereby, the thickness is some 10 to 180 times smaller than the wavelength in the visible band. PRs are the Fabry-Perot-like resonances, formed by the reflections of the short-range surface plasmon wave of the corresponding metal layer from the strip edges [2]. Therefore, their wavelengths can be easily tuned by changing the strip width and thickness. In addition to PRs, multi-element finite gratings made of sufficiently massive silver strips have attractive properties of extraordinarily large reflection, transmission, absorption, and near-field enhancement that are inherited from the light scattering by infinite strip gratings. Namely, these phenomena are greatly effected by the so-called grating resonances that appear due to periodicity $[3,4]$. It should be noted that, until recently, these resonances have been commonly explained via the Rayleigh anomalies [3], and it was only in [4] that the true nature of these specific resonances was explained.

Therefore, in this paper we focus our study on the periodicity-induced resonance effects in the light scattering by finite silver nanostrip gratings located in free space. As a reliable instrument, we use the developed earlier by us median-line integral equation method based on the two-side generalized boundary conditions (GBCs) [5] and Nystrom-type discretization of the interpolation type $[6,7]$. 


\section{Setting the problem and generalized boundary conditions}

Geometry of a flat strip grating made of finite number $N$ of identical silver strips is shown in Fig. 1. Assume that the incident wave is an H-polarized plane wave coming to the grating at the angle $\beta$ from the upper half-space.

The total magnetic field is the sum of the incident wave and the fields scattered by the finite strip grating $H_{z}(\vec{r})=e^{-i k(x \cos \beta+y \sin \beta)}+H_{z}^{s c}(\vec{r})$, where $\vec{r}=(x, y), k$ is the free space wavenumber, and $H_{z}^{s c}(\vec{r})$ is requested to satisfy the Helmholtz equation off the strips contours. Exploiting the small thickness of the strips, $h<<\lambda$, we assume it to be zero, i.e. we shrink their cross-sections to the corresponding median lines $S_{j}=\left\{(x, y): x \in\left[a_{j}, b_{j}\right], y=0\right\}$. This enables us to neglect the internal fields in the strips and impose the two-side GBCs at $S=U_{j=1}^{N} S_{j}$ (see [5, 6] for details),

$$
\begin{aligned}
& \partial\left[H_{z}^{+}(\vec{r})+H_{z}^{-}(\vec{r})\right] / \partial \vec{n}=-i 2 k R\left[H_{z}^{+}(\vec{r})+H_{z}^{-}(\vec{r})\right], \\
& {\left[H_{z}^{+}(\vec{r})+H_{z}^{-}(\vec{r})\right]=-i 2 Q k^{-1} \partial\left[H_{z}^{+}(\vec{r})+H_{z}^{-}(\vec{r})\right] / \partial \vec{n}}
\end{aligned}
$$

Here, the coefficients $R$ and $Q$ are the so-called relative electrical and magnetic resistivities,

$$
\begin{aligned}
& R=i \cot \left(k h \sqrt{\varepsilon_{r}} / 2\right) /\left(2 \sqrt{\varepsilon_{r}}\right), \\
& Q=i \sqrt{\varepsilon_{r}} \cot \left(k h \sqrt{\varepsilon_{r}} / 2\right) / 2,
\end{aligned}
$$

which contain the strip characteristics such as electric thickness $k h$ and relative dielectric function $\varepsilon_{r} ; \vec{n}$ is the unit vector normal to the strip grating; and the superscripts \pm denote the limit values of the field at the top and bottom faces of the strip, respectively. These GBCs are valid if $k h<<1$ and $\left|\varepsilon_{r}\right|>>1[5,6]$. In addition, $H_{z}^{s c}(\vec{r})$ must satisfy the Sommerfeld radiation condition at infinity and condition of the local energy finiteness. Such a scattering problem is uniquely solvable.

\section{Singular and hyper-singular IEs and Nystrom-type discretization}

To satisfy the Helmholtz equation and radiation condition, we seek the scattered field as a sum,

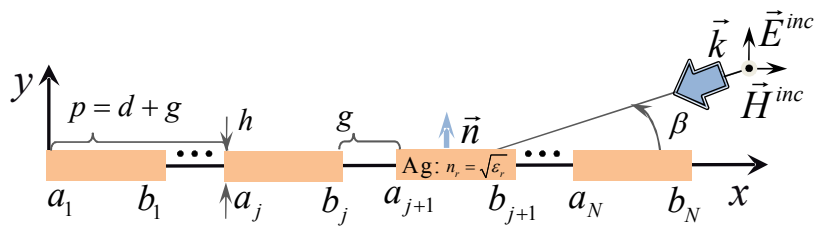

Fig. 1. Geometry of a finite grating of silver nanostrips having the width $d$, thickness $h$ and period $p$.
$H_{z}^{s c}(\vec{r})=\sum_{j=1}^{N}\left[k \int_{S_{j}} v_{j}\left(\vec{r}^{\prime}\right) G\left(\vec{r}, \vec{r}^{\prime}\right) d \vec{r}^{\prime}+\int_{S_{j}} w_{j}\left(\vec{r}^{\prime}\right) \frac{\partial G\left(\vec{r}, \vec{r}^{\prime}\right)}{\partial \vec{r}^{\prime}} d \vec{r}^{\prime}\right]$,

where $G\left(\vec{r}, \vec{r}^{\prime}\right)=(i / 4) H_{0}^{(1)}\left(k\left|\vec{r}-\vec{r}^{\prime}\right|\right) \quad$ is the Green function. Note that the unknown functions $v_{j}(\vec{r}), w_{j}(\vec{r})$ are magnetic and electric currents, respectively, induced on the strips of the grating.

Using GBC (1), (2) and the properties of the limit values of potentials in (4), we obtain two independent sets of $N$ IEs of the second kind. One of them, for all $v_{i}(x), i=1, \ldots, N$, contains equations with logarithmictype singularities, and the other, for $w_{i}(x)$, with hypertype singularities,

$4 Q v_{i}\left(x_{0}^{i}\right)+k \sum_{j=1}^{N} v_{j}(x) H_{0}^{(1)}\left(k\left|x-x_{0}^{i}\right|\right) d x=4 i e^{-i k x_{0}^{i} \cos \beta}$,

$4 R w_{i}\left(x_{0}^{i}\right)+\sum_{j=1}^{N} \int_{a_{j}}^{b_{j}} w_{j}(x) \frac{H_{1}^{(1)}\left(k\left|x-x_{0}^{i}\right|\right)}{\left|x-x_{0}^{i}\right|} d x=4 \sin \beta e^{-i k x_{0}^{i} \cos \beta}$

Note that the integrals in (6) are understood in the sense of finite part of Hadamard. Transforming to new normalized variables $t, t_{0} \in[-1,1]$, introducing new unknown surface functions as $\widetilde{w}_{j}(t)=w_{j}(t)\left(1-t^{2}\right)^{-1 / 2}$ and following $[6,7]$, we isolate the singularities and discretize the resulted sets of $N$ IEs using the Nystromtype method with two different quadrature rules of interpolation type. For IEs (5), we use the GaussLegendre quadrature formulas of the $n_{v}$-th order with nodes in the nulls of Legendre polynomials $P_{n_{v}}\left(\tau_{j}\right)=0$, $j=1, \ldots, n_{v}$. For IEs (6), the use of the Gauss-Chebyshev quadrature formulas of the $n_{w}$-th order (with the weight $\left.\left(1-t^{2}\right)^{1 / 2}\right)$ is more efficient, with nodes in the

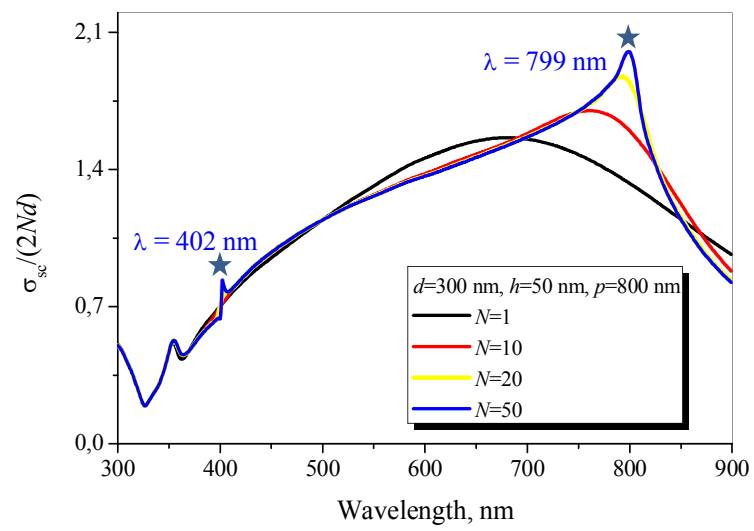

Fig. 2. Normalized TSCS per one strip versus the wavelength for the finite grating of $N$ silver strips with the width $d=300 \mathrm{~nm}$, thickness $h=50 \mathrm{~nm}$, period $p=800 \mathrm{~nm}$ and $\beta=\pi / 2$ (normal incidence) 

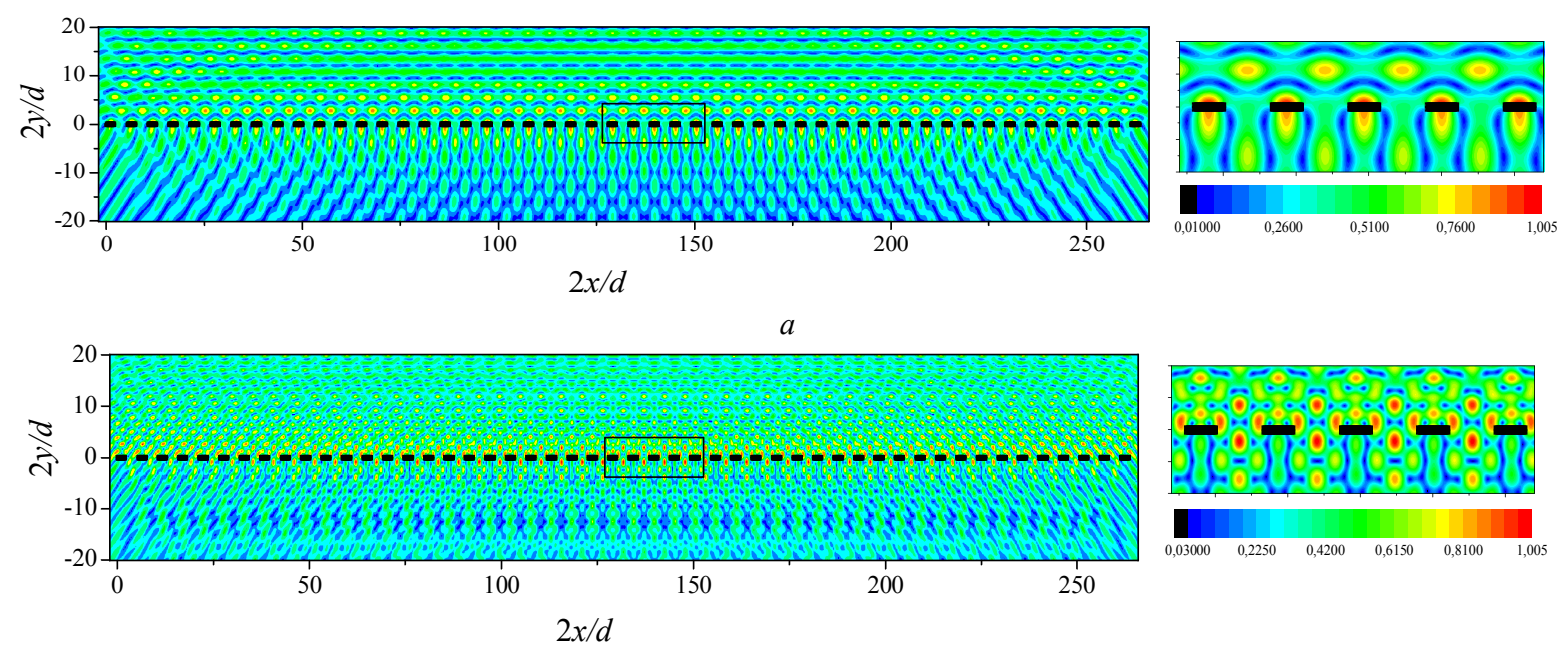

$b$

Fig. 3. Normalized near-field patterns for the plane H-wave $(\beta=\pi / 2)$ normally incident on the grating of $N=50$ silver strips of $d=300 \mathrm{~nm}, h=50 \mathrm{~nm}$ and $p=800 \mathrm{~nm}$ at the wavelengths of the grating resonances $\lambda=799 \mathrm{~nm}$ (a) and $402 \mathrm{~nm}$ (b).

nulls of Chebyshev polynomials of the second kind, $t_{j}=\cos \left(\pi j / n_{w}\right), j=1, \ldots, n_{w}$. In view of the limited space, we omit the details and refer to [6-8]. Thereby, applying the above mentioned quadrature formulas, we arrive at two independent sets of matrix equations of the orders $N \cdot n_{v}$ and $N \cdot n_{w}$, respectively, for the values $v_{j}\left(\tau_{i}\right)$ and $\widetilde{w}_{j}\left(t_{k}\right)$. These matrix equations represent discrete models of our IEs. On solving them we obtain the surface currents as interpolations polynomials.

The chosen quadrature formulas ensure rapid convergence of numerical solutions to the accurate ones if $n_{v}, n_{w} \rightarrow \infty$. Conservative estimation gives the rates of convergence as $O\left(1 / n_{v, w}\right)$, although the actual rate is always higher $[6,7]$. The empiric rule to achieve 4-digit accuracy in the analysis of surface currents is to take $n_{w}=k d \cdot \alpha_{S R P W}+5$ and $n_{v}=k d+5$ where $\alpha_{\text {SRPW }}$ is the effective refractive index of short-range surface plasmon wave of the corresponding metal layer $[6,8]$. For instance, for a $20-\mathrm{nm}$ thick and $2 \lambda$-wide silver strip in the whole visible range, one can take $n_{v}=n_{w}=50$.

\section{Numerical simulation: plasmon and grating resonances}

To study the plasmon and periodicity resonance effects in the H-polarized light scattering by the finite silver nanostrip gratings, we have investigated the wavelength dependences of the total scattering cross section (TSCS), obtained via integration of the far-field scattering pattern, and visualized the near-field patterns at resonance wavelengths. To characterize the complex dielectric permittivity of silver, we took the experimental data of Johnson and Christy with spline interpolation.
As one can see from Fig. 2, a stand-alone silver strip with $h=50 \mathrm{~nm}$ has two PRs in the visible range at the normal incidence $(\beta=\pi / 2)$ : at $\lambda=354.1 \mathrm{~nm}$ and $\lambda=$ $680.5 \mathrm{~nm}$. The TSCS of silver grating of $N=50$ strips exhibits, after normalization by $N$, two types of resonances: PRs and GRs (the latter resonances are marked with asterisks).

Here, the first G-resonance wavelength is $\lambda=$ $402 \mathrm{~nm}$ that is slightly above $p / 2$, and another one at $\lambda=799 \mathrm{~nm}$ that is close to $p$. In fact, within the whole range between 600 and $850 \mathrm{~nm}$, a combined resonance takes place; such merging leads to considerable enhancement of scattering per a strip of the grating.

Presented in Fig. 3 are the total near-field patterns at the corresponding wavelengths, $\lambda=799 \mathrm{~nm}$ (a) and $\lambda=402 \mathrm{~nm}$ (b), together with their zooms around five central periods.

One can see two different standing waves: one is formed at $y>0$, because of enhanced reflection, and another one stands along the $x$-axis, formed by the quasiFloquet harmonics of the grating. To deepen the understanding of the resonance effects, in Fig. 4 we show the reliefs of TSCS versus two parameters: wavelength and grating period while $d=300 \mathrm{~nm}$ (a) and wavelength and grating width while $p=800 \mathrm{~nm}$ (b) for the grating of $N=20$ silver strips under the normal incidence.

In Fig. 4a, a pronounced sharp "ridge" stretching along the line $\lambda=p$ is observed and a smaller one is discernible along $\lambda=p / 2$. They correspond to the mentioned above GRs and run across the broad "hill" of the main PR of the given strip-width. For the finite strip grating with a fixed period of $800 \mathrm{~nm}$ (Fig. 4b), under the normal incidence, two PR "hills" are cut by two pronounced GR "ridges" at almost fixed wavelengths near $\lambda \approx p / 2$ and $\lambda \approx p$; these GRs are best visible in the case of the strip width from 100 to $200 \mathrm{~nm}$ and from 300 to $500 \mathrm{~nm}$, respectively. 

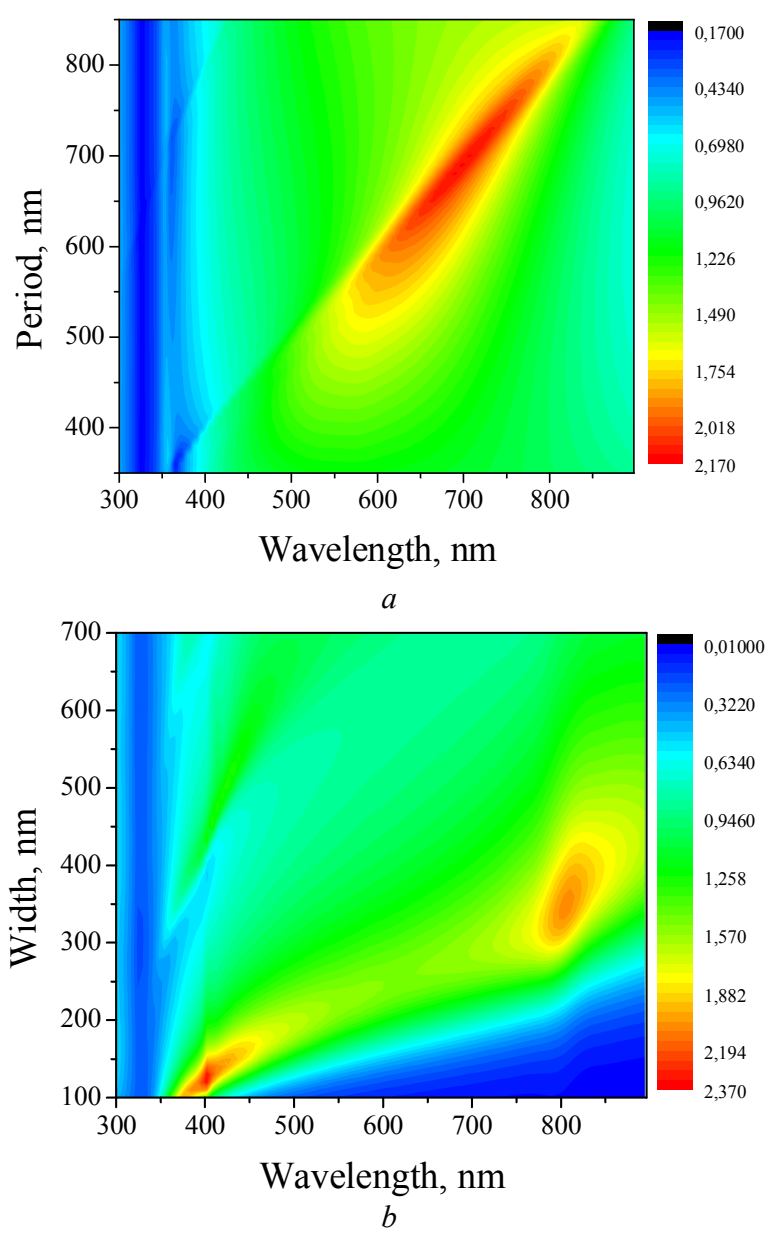

Fig. 4. Normalized per one strip reliefs of TSCS (in nm) versus the wavelength and the period while $d=300 \mathrm{~nm}$ (a) and versus the wavelength and width while $p=800 \mathrm{~nm}$ (b) for the silver strip grating of $N=20$ of thickness $h=50 \mathrm{~nm}$ under the normal incidence $(\beta=\pi / 2)$.

\section{Conclusions}

Summarizing, we have studied the scattering and absorption of the H-polarized electromagnetic wave by free-standing finite silver nanostrip gratings, in the visible range. The analysis has shown co-existence of the localized surface-plasmon resonances of a standalone silver strip and the grating resonances close to $\lambda \approx p / m, m=1,2, \ldots$ caused by the effect of periodicity. Here, the gratings of the larger numbers of strips have more pronounced grating resonances. The effect of enhanced scattering in the combined resonance has been revealed.

\section{Acknowledgements}

This work has been partially supported by the National Academy of Sciences of Ukraine via the State Target Program "Nanotechnologies and Nanomaterials" and the European Science Foundation via the Research Networking Programme "Newfocus".

\section{References}

1. V. Giannini and J.A. Sànchez-Gil, Calculations of light scattering from isolated and interacting metallic nanowires of arbitrary cross section by means of Green's theorem surface IEs in parametric form // J. Opt. Soc. Am. A, 24(9), p. 241-248 (2007).

2. T. Søndergaard and S.J. Bozhevolnyi, Strip and gap plasmon polariton optical resonators // Phys. Status Solidi (b), 245(1), p. 9-19 (2008).

3. A. Christ, T. Zentgraf, J. Kuhl, S.G. Tikhodeev, N.A. Gippius, and H. Giessen, Optical properties of planar metallic photonic crystal structures: experiment and theory // Phys. Rev. B, 70(12), 125113-125128 (2004).

4. D.M. Natarov, V.O. Byelobrov, R. Sauleau, T.M. Benson, and A.I. Nosich, Periodicity-induced effects in the scattering and absorption of light by infinite and finite gratings of circular silver nanowires // Opt. Express, 19(22), 22176-22190 (2011).

5. K.M. Mitzner, Effective boundary conditions for reflection and transmission by an absorbing shell of arbitrary shape // IEEE Trans. Antennas Propag., 16(6), p. 706-712 (1968).

6. O.V. Shapoval, R. Sauleau, and A.I. Nosich, Scattering and absorption of waves by flat material strips analyzed using generalized boundary conditions and Nystrom-type algorithm // IEEE Trans. Antennas Propag., 59(9), p. 3339-3346 (2011).

7. M.V. Balaban, E.I. Smotrova, O.V. Shapoval, V.S. Bulygin, and A.I. Nosich, Nystrom-type technique for solving electromagnetics integral equations with smooth and singular kernels // Int. J. Numerical Modeling: Electronic Networks: Devices and Fields, 25(DOI: 10.1002/jnm.1827) (2012).

8. O.V. Shapoval, R. Sauleau, and A.I. Nosich, Coplanar gratings of multiple thin noble-metal nano-strips as surface plasmon resonance structures // Proc. Int. Conf. Transparent Optical Networks (ICTON-11), Stockholm, We.B2.4 (2011). 